\title{
An Adaptive Scheduling Algorithm for Bluetooth Ad-hoc Networks
}

\author{
Raymond Y.L. Lee and Vincent W.S. Wong \\ Department of Electrical and Computer Engineering \\ The University of British Columbia,Vancouver, BC, Canada \\ e-mail: \{rayl, vincentw $\}$ aece.ubc.ca
}

\begin{abstract}
In this paper, we propose an Adaptive Scheduling Algorithm (ASA) for Bluetooth scatternets. ASA is adaptive in which the bandwidth allocated on each link or session is dynamically adjusted based on the estimated traffic. ASA integrates both intra-piconet and inter-piconet scheduling to improve the aggregate throughput and delay. ASA prevents the bridge node conflict and satisfies the max-min fairness criterion. We compare our proposed ASA with two other scheduling algorithms via simulations. Results show that ASA provides good performance in terms of fairness, aggregate throughput, and average delay.
\end{abstract}

\section{INTRODUCTION}

Bluetooth [1] is a short-range wireless communications technology. It operates in the $2.4 \mathrm{GHz}$ unlicensed Industrial Scientific Medical (ISM) band. The ISM band is divided into 79 frequency sub-bands. A channel in Bluetooth is defined by a pseudo-random frequency hopping sequence hopping through the 79 frequency sub-bands at a rate of 1600 hops per second. The result is a slotted channel with the slot duration equal to $625 \mu \mathrm{s}$. The raw data rate is defined as $1 \mathrm{Mbps}$.

Bluetooth devices are required to form a piconet before exchanging data. Each piconet has a master unit that controls the channel access and frequency hopping sequence. Other nodes in the piconet are referred to as the slave units. Within a piconet, there is one master and up to seven active slaves, and all devices share the same wireless channel. In order to minimize the interference between different piconets, the frequency hopping sequence is unique for each piconet.

In a piconet, the master controls the access of all devices to the channel through a time division duplex master-slave polling scheme. Bluetooth supports different traffic types. For voice traffic, the master maintains a Synchronous ConnectionOriented (SCO) link by reserving slots at regular intervals. For data traffic, the master can establish an Asynchronous Connection-Less (ACL) link on slots not reserved for SCO links. Bluetooth defines different packet types. A packet can occupy either 1, 3, or 5 time-slots. Packets with forward error correction capability (FEC) are identified as DM1, DM3, and DM5. Packets without FEC are identified as DH1, DH3, and DH5. For communication between a master and its slave, the master begins by sending either a data packet or a POLL packet to the slave. The slave will then reply by sending either a data packet or a NULL packet.

Several piconets can be interconnected via bridge nodes to create a scatternet. Bridge nodes are capable of time-sharing between multiple piconets, receiving packets from one piconet and forwarding them to another. A bridge node can be a master in one piconet and act as slave in other piconets. This is called a master/slave bridge. Alternatively, a bridge node can act as a slave in all the piconets it is connected to. This is
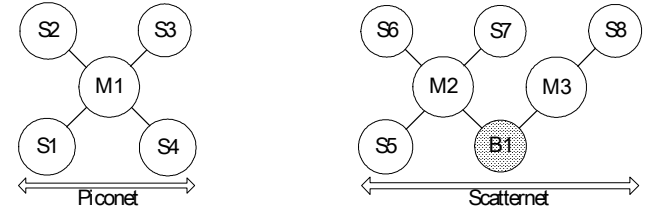

Fig. 1 Piconet and scatternet network

called a slave/slave bridge.

Scheduling in Bluetooth scatternets can be divided into two tasks, namely: intra-piconet scheduling and inter-piconet scheduling. Intra-piconet scheduling focuses on the scheduling of packets transmission within a piconet. Since a slave node cannot transmit a packet without first being polled by its master, intra-piconet scheduling is controlled by the master. On the other hand, inter-piconet scheduling focuses on when a bridge node switches between piconets and how a bridge node communicates with the masters in different piconets.

An efficient intra-piconet scheduling algorithm should minimize the number of wasted slots, assign time-slots dynamically based on the traffic rate, and maintain fairness among different slaves. Since each master-slave polling operation includes the transmission from the master to its slave and a reply from the slave to its master, an empty queue in either side will result in a wasted slot. The master has limited knowledge on the buffer occupancy of its slaves. Each slave may have different bandwidth requirement.

The main issue in inter-piconet scheduling is the switching of a bridge node between piconets. Since each Bluetooth device has one transceiver, it can only participate in one piconet at a time. As each master uses its own local clock, a bridge node has to re-synchronize with the new master when it switches to a new piconet. The switch between two piconets may result as a slot loss. Another problem occurs when two masters try to access the bridge node simultaneously. This is referred to as the bridge node conflict. Since a bridge node can only listen to one master at a time, the other master will not be able to communicate with the bridge node and will waste slots for the polling operation. Lastly, an efficient inter-piconet scheduling scheme needs to dynamically allocate bandwidth on each link and maintain fairness among all nodes.

In this paper, we propose an Adaptive Scheduling Algorithm (ASA) that focuses on achieving max-min fairness for all nodes within the scatternet, preventing bridge node conflict, and dynamically allocating bandwidth for each link based on traffic changes. As a result, it leads to improving performance in aggregate throughput and average delay.

The rest of this paper is organized as follows. In Section II, we discuss the previous work on scatternet scheduling. We describe the ASA in Section III, and evaluate its performance by comparing with two other schemes in Section IV. Section 
V concludes our work.

\section{RELATED WORK}

In this section, we describe the related work on interpiconet scheduling. In [2], Tassiulas et al. propose a service token allocation scheme for scheduling. Tokens are generated at the max-min fair rate. However, the scheme is not fully distributed since the matching operation is centralized. In [3], Johansson et al. propose the use of the sniff mode to assign rendezvous point (RP). The distance between two RPs limits the time for a bridge node to stay in a piconet. Since the scheme uses fixed bandwidth allocation, it may be inefficient for real-time dynamic traffic. In [4], Zhang and Cao propose the flexible scatternet-wide scheduling (FSS) scheme, which places an adjustable switch table on each bridge node and master. Both the bridge node and the master know when they can communicate with each other. Since the scheme gives a higher priority for traffic on bridge node, it may not maintain fairness for all the nodes. Johansson et al. propose the JUMP mode in [5], which allows a bridge node to choose the time to switch between piconets. In [6], Shai et al. propose a load adaptive algorithm, which utilizes decision variables to determine the time period for a bridge node to stay in each piconet. Although the time for a bridge node to spend on a piconet can be adapted to traffic change, this scheme focuses on small-scale scatternets (which just involves two piconets).

In [7], Kapoor et al. propose a distributed scatternetscheduling algorithm, which allocates bandwidth to each link based on traffic estimation. Each time when a master meets with a bridge node, they will negotiate their next meeting time based on local traffic estimation. However, in a dynamic environment, it is difficult to predict accurately the future traffic. A traffic estimation error will affect the performance. Tan et al. in [8] propose a locally coordinated scheduling scheme, which schedules the meeting time between two nodes based on traffic conditions. Each time before a node terminating the meeting with the connected node, they will negotiate the start time and the duration time for the next meeting. Nevertheless, the scheme does not consider fairness as a factor for scheduling. In [9], Baatz et al. propose a creditbased scheduling (CBS) scheme, which focuses on fair link bandwidth allocation in a node. Each node assigns credits to each of the connected link. The credits are allocated according to link utilization. Since each node makes its own decision to communicate with other node according to local share of bandwidth, the bridge node conflict may occur sometimes. In [10], Raez et al. propose a scatternet scheduling algorithm, which utilizes a pseudo random sequence to define the start time for all the meetings between two nodes. In addition, individual node will skip some of the meeting time based on traffic change. However, since two nodes do not guarantee to meet at the same time, it may result as a miss of meeting time between two nodes.

\section{ADAPTIVE SCHEDUling AlgORITHM (ASA)}

We make the following assumptions in our work [11]: (1) a bridge node is a slave-slave node; (2) only ACL connections are present in the scatternet; (3) all nodes are time-

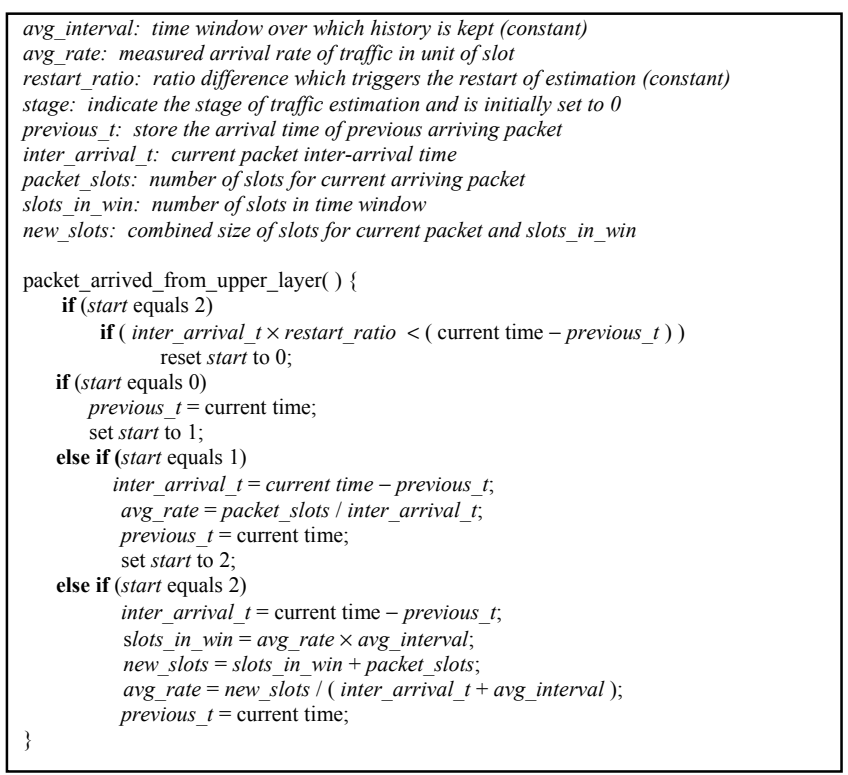

Fig. 2 Algorithm for traffic rate estimation

synchronized with each other within the scatternet.

In order to prevent bridge node conflict and at the same time provide max-min fairness for all the links within the scatternet, each master node or bridge node maintains a dynamic switch schedule to organize the time for it to communicate with each connected node. A bandwidth allocation around a node is said to be max-min fair if a link cannot increase its bandwidth without decreasing that of any other link receiving less or equal bandwidth [13]. In addition, a master node also maintains an active list and a waiting list to organize the serving time for each slave node. Furthermore, an estimator is placed on each node for real-time traffic monitoring.

\section{A. Traffic Estimation}

ASA uses a time-sliding window (TSW) [14] for traffic estimation. The TSW maintains a time-based history and can obtain a smooth average for bursty traffic.

Fig. 2 shows the algorithm for the traffic estimation. The algorithm uses the first two packets to calculate the initial packet arrival rate. It then updates the estimated packet arrival rate by using the TSW scheme. Since a long idle period will affect the accuracy of traffic estimation, the traffic estimator restarts the estimation when the new packet inter-arrival time is larger than the previous value by a predefined restart_ratio. The value of restart_ratio in ASA is set to 5 .

Besides estimating the packet arrival rate, each node also needs to determine the time for it to accumulate enough packets request for data transmission.

In order to maintain fairness for all nodes within the scatternet, ASA defines the maximum usable serving slots (MUSS) to limit the serving time between two nodes. The size of MUSS must be large enough for both uplink and downlink transmissions. If the scatternet supports 1-slot, 3-slots and 5slots packets, then the size of MUSS must be larger than or 


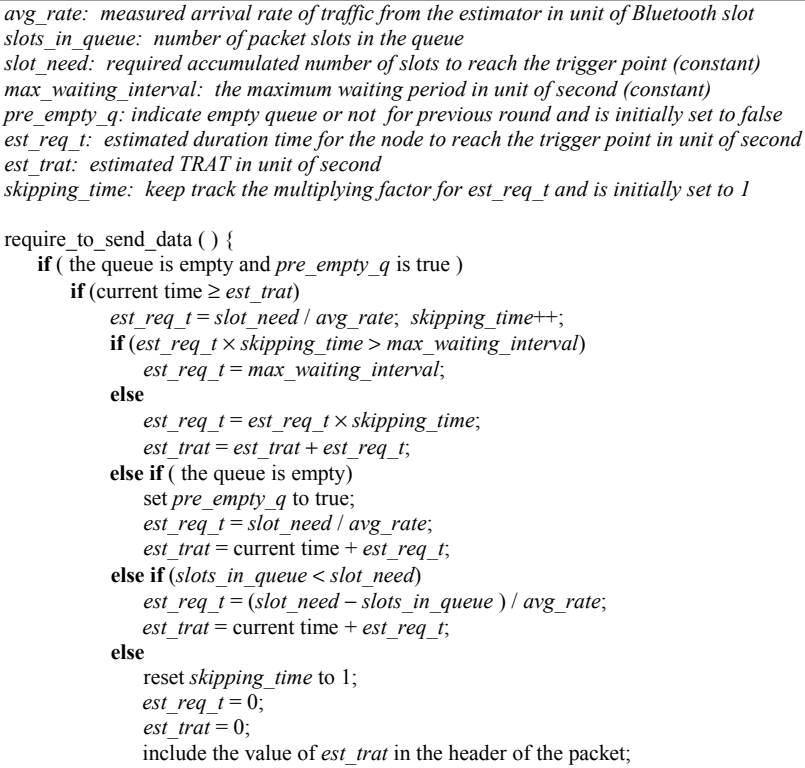

Fig. 3 Algorithm for updating estimated time to reach the trigger point

equal to ten Bluetooth time slots. The trigger point for oneway transaction of a node is defined as half the size of MUSS. In ASA, the time for a node to reach the trigger point is defined as the transmission request arrival time (TRAT).

Fig. 3 shows the algorithm for how each node updates the estimated TRAT before it transmits a packet. If a node already has enough packets in the queue, it will indicate that no waiting time is needed. Otherwise, it will use the estimated packet arrival rate to predict the TRAT. As a result, if a node already has enough packets in the queue, ASA does not require the traffic estimation information to determine the status of the node. In order to reduce the number of wasted slots during an idle period, ASA maintains a variable called skipping time to modify the waiting time. For each consecutive occurrence of an empty queue, if the current time has already passed the estimated TRAT of the node, the node will increase the value of the skipping time by one. ASA defines the maximum waiting interval to limit the waiting time for the node to request for data transmission. If the value of skipping time multiplies the original estimated duration time required for the node to reach the trigger point is smaller than the maximum waiting interval for the node, it will be set as the new duration time. Otherwise, the maximum waiting interval will be set as the next duration time for the node to reach TRAT. Once the node accumulates enough packets to reach the trigger point, ASA re-sets the value of skipping_time to 1 .

\section{B. Intra-Piconet Scheduling}

In ASA, each master node maintains an active list and a waiting list to schedule the serving order for all connected slave nodes. An active list contains all the slave nodes that have accumulated enough packets to reach the trigger point. A waiting list contains all the slave nodes that do not have enough packets in the queue. The master follows the order of nodes in its active list and serves them in a round robin

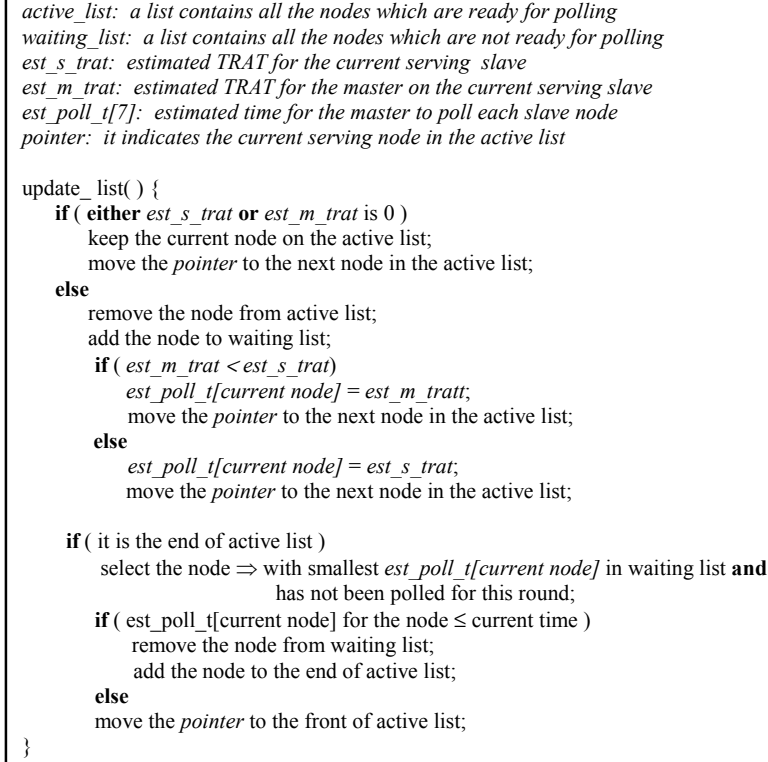

Fig. 4 Algorithm for updating active and waiting list

fashion. The master starts to serve the next slave node either when it has finished serving the current slave node for MUSS or when there is no data to send between the two nodes. A master uses the dynamic switch schedule to reserve a fixed size switch schedule slot (SS_Slot), which is equal to the size of MUSS

Sometimes two nodes may spend less than MUSS for data transmission. In that case, there will be some slots available before the master encounters an SS Slot assigned to a bridge node. The master will continue to serve the next node in the active list until the time for the master to communicate with a bridge node occurs. Before the master serves another node, it will update the status of the slave node in the active list.

Fig. 4 shows the algorithm for updating the active and waiting lists. When the master encounters an empty SS Slot, it begins to serve the slave node in the active list. Each time when a slave node replies the packet to the master, it indicates its estimated TRAT in the packet's header. The master will only use the information to update the active list when it switches to serve another node. At that time, if either a master or a slave indicates that it has already accumulated enough packets to reach the trigger point, the master will keep the slave in the active list. Otherwise, the slave will be moved to the waiting list. The master also stores the estimated time for it to poll the slave again. The estimated polling time is chosen from the smaller time between the estimated TRAT for the master and the estimated TRAT for the slave. If the master reaches the end of the active list, it will select a slave node which has the smallest estimated polling time and has not been polled for this round in the waiting list.

ASA can maintain the max-min fairness at the master node based on the following reasons. Once a link between a slave and the master has accumulated enough packets to reach the trigger point, the slave node is guaranteed to be in the active list in the current round and to be served by the master with MUSS once in a around. In addition, in each round, the 


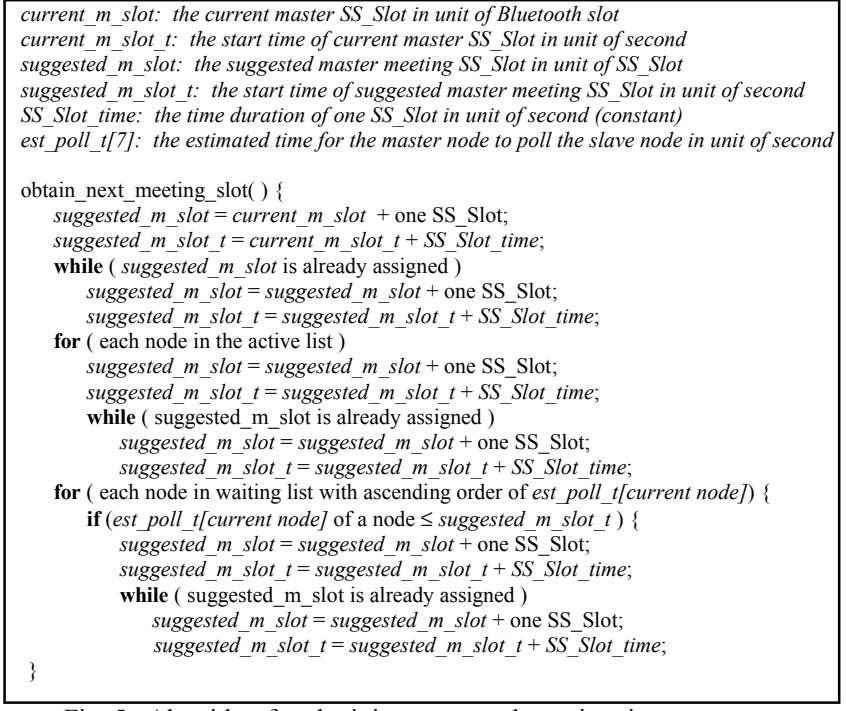

Fig. 5 Algorithm for obtaining suggested meeting time on master

maximum number of links served by a master is equal to the total number of links connected to the master. Therefore, if a link generates traffic at a rate lower than or equal to the equal shared bandwidth, the link will not demand for more than one MUSS in a round. As a result, the link will always be satisfied. In addition, links with the same traffic rate will be added to the active list with the same number of times. As a result, they will receive the same amount of bandwidth.

\section{Inter-piconet Scheduling}

A switch schedule is maintained between a master and a bridge node to organize the time for them to communicate with each other. Each time when a master meets with a bridge node, they negotiate their next meeting time and update their switch schedule. In ASA, a master uses the hold mode to allow the bridge node to switch between piconets. In hold mode, a master puts a connected node into sleep state in which the node does not require to listen to the master for a period of time. When the time expires, the connected node switches to an active node and actively listens to the master. Therefore, after determining the new meeting time, a master node knows how long it should put a bridge node into hold mode. The negotiation process is initialized by the master.

Fig. 5 shows the algorithm for how the master node finds the suggested next meeting time. The master node first finds an empty SS_Slot as the initial estimated meeting time. The size of an SS_Slot is equal to the size of MUSS. Then for each slave node in the active list, the master will reserve an empty SS_Sot for it to indicate that the SS_Slot is not eligible for scheduling with a master-bridge meeting time. Moreover, the master will skip the SS_Slot that has already been assigned to a link. The process continues until the master reaches the end of the active list.

After reserving SS Slots for the active nodes, the master also checks the nodes in the waiting list. Thus, starting from the node with the earliest estimated polling time in the waiting list, if the start time for current estimated meeting SS_Slots is larger than the node's estimated polling time, the master will

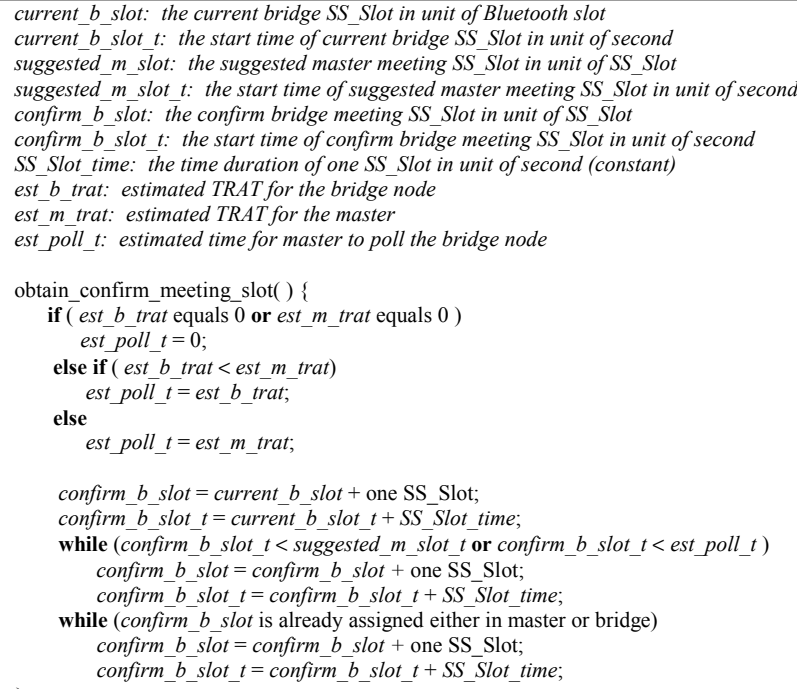

Fig. 6 Algorithm for obtaining confirmed meeting time on bridge node

reserve an empty SS_Slot for the node. The process will not end until the master finishes checking all the nodes in the waiting list. As a result, the master can determine the final suggested meeting time with the bridge node, which is located at the start time of the estimated meeting SS Slot. Thus, at the beginning of each meeting time, the master will send the estimated meeting SS_Slot, the estimated TRAT, and the location for all the assigned SS_Slots to the bridge node for new meeting time negotiation. In addition, the master switches to serve another node either when it has finished serving the bridge node for MUSS or when there is no data to send between the two nodes. Therefore, by reserving SS_Slot for nodes in the active list, it can maintain max-min fairness for all the nodes within the piconet.

Once the bridge node receives the information from the master, it uses the information together with its local estimation values to determine the confirmed meeting time. Fig. 6 shows the algorithm for the process. At the beginning, the bridge node finds the time for both nodes to fulfill the trigger point requirement. Thus, if both nodes have already reached the trigger point, the bridge node will indicate that no waiting time is needed. However, if both nodes indicate that they do not have enough packets to reach the trigger point, the bridge node will select the smaller estimated TRAT between the master and bridge node as the final estimated TRAT. Afterwards, the bridge node finds the closest SS_Slot with a start time exceeding both the final estimated TRAT and the start time of meeting SS_Slot suggested by the master. If the SS_Slot has already been assigned to a link, the bridge node will find the next closest empty SS_Slot. As a result, the bridge node assigns the empty SS_Slot to the master node, and selects the start time of the SS_Slot as the confirmed meeting time. Finally, the bridge node includes the confirmed meeting SS Slot in the packet reply to the master. The master then assigns the confirmed meeting SS_Slot to the bridge node.

ASA achieves max-min fairness around a bridge node for the following reasons. By not serving the bridge node until the 


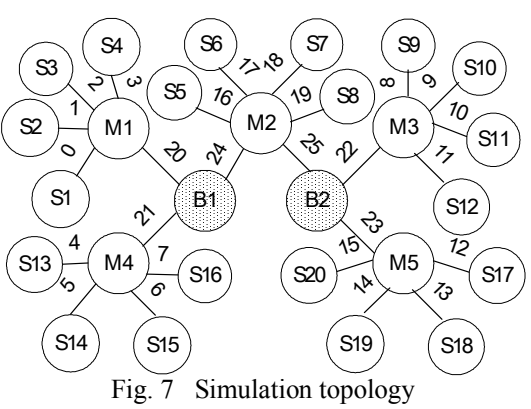

start time of confirmed meeting SS_Slot, it is analogous to placing the bridge node in the waiting list when it is not ready to receive service. The residual bandwidth will be re-allocated to other links around a bridge node by allowing them to reserve an SS_Slot before the confirmed meeting SS_Slot. Since a master serves a bridge node for MUSS at their confirmed meeting SS_Slot, it is similar to serving the node with equal bandwidth when the node is added back to the active list. In addition, when a master suggests a meeting time, it maintains fairness around itself by reserving SS_Slots for nodes in active list. When a bridge node confirms the meeting time with the master, it maintains fairness around itself by ensuring the link will accumulate enough packets to reach the trigger point at the meeting time. Lastly, by skipping SS_Slot that has already been assigned to a link, ASA can prevent bridge node conflict within a scatternet.

\section{Resume Send}

Since the time-slots available may not always be enough for a particular packet size for transmission (e.g., DM5), ASA implements the resume send mode. The master updates the connected node (i.e., slave) with the remaining time-slots available. The slave updates the master with the size of packet waiting in its queue. If the remaining slots are too small for the packet transmission, the slave will activate resume send mode and indicate it by sending a null packet. If the master notices that the remaining slots are not enough for packet transmissions in its queue but is enough for packet transmission in the slave's queue, the master will send a poll packet to slave.

\section{PERformance EVAluAtion}

We compare the performance of our proposed ASA with CBS [9] and FSS [4] via computer simulations [15]. Fig. 7 shows the scatternet topology.

\section{A. Fairness Comparison with Constant Bit Rate Traffic}

We assume that there is only bi-directional traffic between each master and its connected nodes (both pure slaves and slave/slave bridge nodes). No traffic runs across a bridge node. All traffic streams generate constant bit rate traffic and DH1 packets are used. We assume that each sender always has packets to send. When we ignore the packet collisions due to interference from neighboring piconets, the maximum aggregated throughput in each piconet is $27 \times 8 / 625 \times 10^{-6}=$ $345.6 \mathrm{kbps}$. In Fig. 7, since masters M1, M3, M4 and M5 have five links each, the ideal max-min bandwidth sharing for each link is $1 / 5 \times 345.6 \mathrm{kbps} \cong 69 \mathrm{kbps}$. Since master M2 has six links, the ideal max-min bandwidth sharing for each link is $1 / 6 \times 345.6 \mathrm{kbps} \cong 58 \mathrm{kbps}$. The simulation time for each run is 60 s. Fig. 8 (a) to (c) shows the throughput distribution for each scheme with the ideal max-min fair bandwidth allocation. It shows that ASA achieves the same result as with the ideal values. However, since FSS focuses on master-bridge traffic, it does not allocate fair amount of bandwidth to master-slave links. Lastly, since CBS does not prevent bridge node conflict, it leads to a lower bandwidth allocation on the master-bridge links. Note that if we include the packet collisions in our simulations, the aggregate throughput will be lower than the ideal values. However, the relative performance between ASA, CBS, and FSS remains the same.

\section{B. Comparison with UDP (User Datagram Protocol) Traffic}

In this simulation, we consider bursty on-off UDP traffic. The on and off periods follow exponential distributions with an average length of $1 \mathrm{~s}$ and $2 \mathrm{~s}$, respectively. The UDP traffic is encapsulated in DH1 packets. We consider the following bidirectional traffic flows: M1-M2, M2-M4, M4-M1, M2-M3, M3-M5, and M5-M2. Moreover, we also consider traffic between each master and its slaves. In the first set of simulation, each sender in piconet of M1, M3, M4, and M5 generates packets at a rate of $34.5 \mathrm{kbps}$ and each sender in piconet of M2 generates packets at a rate of $29 \mathrm{kbps}$. We choose these rates by dividing the max-min bandwidth share for the link by half. We then vary the traffic rate by a factor of $0.6,1.5,1.8,2,2.4,2.8$, and 3 to observe the performance change. Fig. 8 (d) and (e) show the aggregate throughput and average delay for all the three schemes. Fig. 8(d) shows that ASA achieves a higher throughput than the other two schemes especially when the traffic rate is high. Fig. 8(e) shows that ASA maintains a lower delay than the other two schemes.

\section{Comparison with TCP Traffic}

In this section, we compare the average end-to-end transfer delay for sending a file from one node to another node using TCP. In the first scenario, only master nodes transmit data. Thus, each master sends a file to each of its slaves. We refer this as the M-S traffic. Moreover, masters M0, M1, M3, and M4 each sends a same size file through a bridge node to M2. We refer this as the M-B-M traffic. We vary the file size from $0.1 \mathrm{MB}$ to $0.5 \mathrm{MB}$ to observe the performance change. Fig. 8(f) shows that ASA has the lowest average transfer delay on M-S traffic while FSS has the highest delay. Fig. 8(g) shows that ASA and CBS have similar performance for the average transfer delay on M-B-M traffic, while FSS has a better performance. This is due to the fact that FSS gives a higher priority on master-bridge link.

In the next scenario, S4, S16, S12, and S20 are removed from the topology in Fig. 7. Only slave nodes transmit data. We setup one-way TCP traffic flows on S1-M1-S2, S9-M3S10, S13-M4-S14, and S17-M5-S18. We refer this as the SM-S traffic. We also setup one-way TCP traffic flows on S3M1-B1-M2-S5, S15-M4-B1-M2-S6, S11-M3-B2-M2-S7, and S19-M5-B2-M2-S8. We refer this as the S-M-B-M-S traffic. Fig. 8 (h) and (i) show that ASA has the lowest average transfer delay for both S-M-S and S-M-B-M-S traffic. 

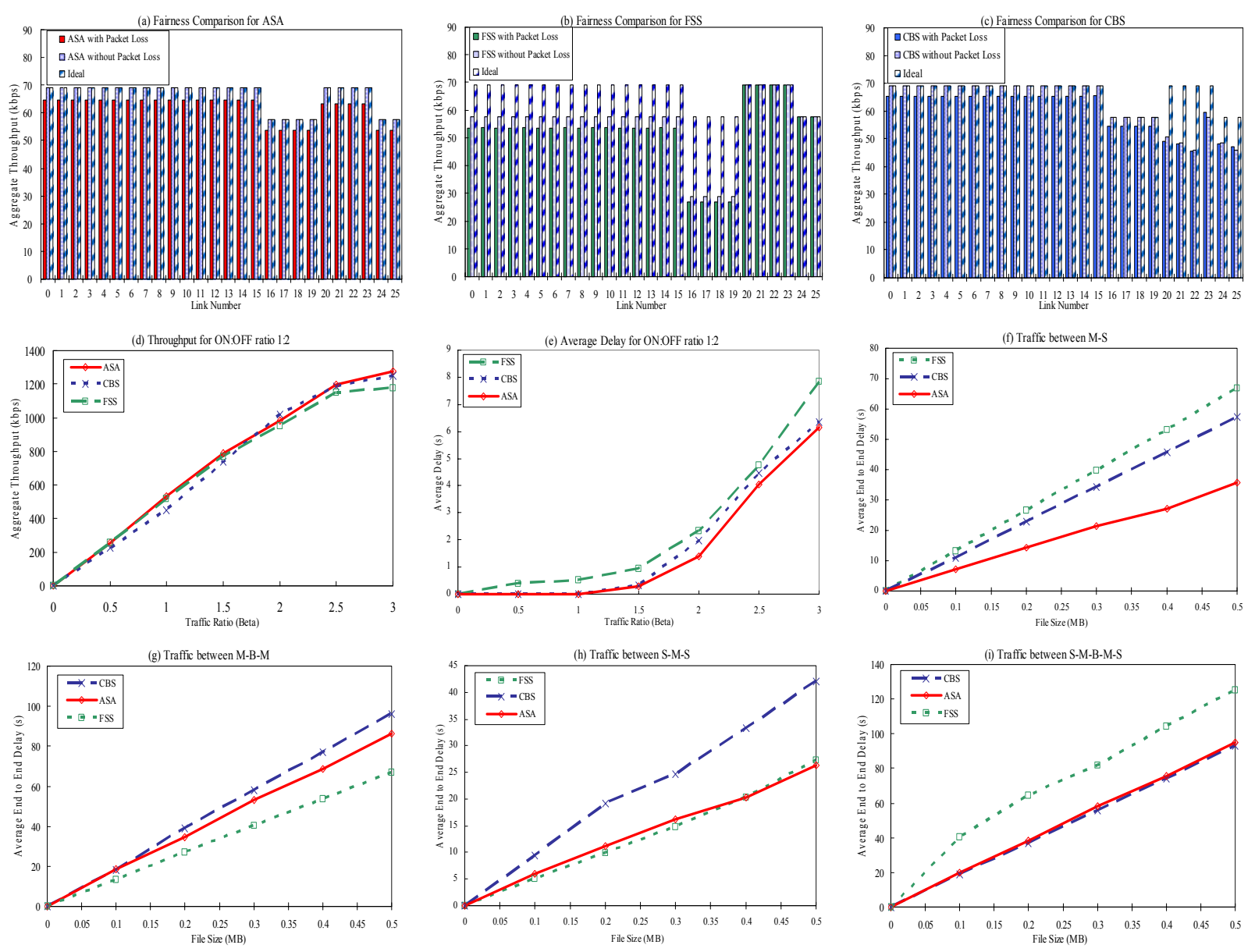

Fig. 8 Performance comparisons among ASA, CBS, and FSS.

\section{CONCLUSIONS}

We proposed an Adaptive Scheduling Algorithm (ASA) for Bluetooth scatternets. ASA can dynamically allocate bandwidth to each link based on traffic change and maintain max-min fairness for all the nodes within the scatternet. Besides, ASA can also prevent the bridge node conflict. Simulation results showed that ASA can maintain a high aggregate throughput and low delay on either CBR or bursty on-off UDP traffic when compared with FSS and CBS. Results also show that ASA can maintain a small average transfer delay for TCP traffic when compared with FSS and CBS. For future work, we plan to study the effects of packet collisions due to interference and fading on scheduling.

\section{ACKNOWLEDGEMENT}

This work was supported by the Natural Sciences and Engineering Research Council of Canada (NSERC) under grant number STPGP 257684-02.

\section{REFERENCES}

[1] Specification of the Bluetooth System - version 1.2, November 2003. Available at "http://www.bluetooth.com".

[2] L. Tassiulas and S. Sarkar, "Maxmin Fair Scheduling in Wireless Networks", in Proc. of IEEE INFOCOM'02, New York, NY, June 2002.

[3] P. Johansson, R. Kapoor, M. Kazantzidis, and M. Gerla, "Rendezvous
Scheduling in Bluetooth Scatternets," in Proc. of IEEE ICC'02, New York, NY, May 2002.

[4] W. Zhang, and G. Cao, "A Flexible Scatternet-wide Scheduling Algorithm for Bluetooth Networks," in Proc. of IEEE IPCCC, Phoenix, AZ, April 2002.

[5] N. Johansson, F. Alriksson, and U. Jonsson, "JUMP Mode - A Dynamic Window-based Scheduling Framework for Bluetooth Scatternets," in Proc. of ACM MobiHoc'01, Long Beach, CA, Oct. 2001.

[6] L. H. Shai, R. Kofman, A. Segall, and G. Zussman, "Load-Adaptive Inter-Piconet Scheduling in Small-Scale Bluetooth Scatternets," IEEE Communications Magazine, vol. 42, no. 7, pp. 136-142, July 2004.

[7] R. Kapoor, A. Zanella, and M. Gerla, "A Fair and Traffic Dependent Scheduling Algorithm for Bluetooth Scatternets," ACM J. Mobile Networks and Applications, vol. 9, no. 1, Feb. 2004.

[8] G. Tan and J. Guttag, "A Locally Coordinated Scatternet Scheduling Algorithm," in Proc. of IEEE LCN, Nov. 2002.

[9] S. Baatz, M. Frank, C. Kuhl, P. Martini, C. Scholz, "Adaptive Scatternet Support for Bluetooth using Sniff Mode," in Proc. of IEEE Conference on Local Computer Networks, Tampa, FL, Nov. 2001.

[10] A. Raez, G. Miklos, F. Kubinszky, and A. Valko, "A Pseudo Random Coordinated Scheduling Algorithm for Bluetooth Scatternets," in Proc. of ACM MobiHoc'01, Long Beach, CA, Oct. 2001.

[11] R. Lee, "An Adaptive Scheduling Algorithm for Bluetooth Ad-hoc Networks," M.A.Sc.'s thesis, University of British Columbia, 2004.

[12] J. Misic, and V. B. Misic, "Bridges of Bluetooth County: Topologies, Scheduling, and Performance," IEEE Journal on Selected Areas in Communications, vol. 21, no. 2, Feb. 2003.

[13] D. Bertsekas and R. Gallager, Data Networks, Prentice Hall, 1992.

[14] D. D. Clark and W. Fang, "Explicit Allocation of Best-Effort Packet Delivery Service," IEEE/ACM Trans. on Networking, Aug. 1998.

[15] "Network Simulator (NS-2)," http://www.isi.edu/nsnam/ns/. 